\title{
Review of: "Biochemical characterisation of human transglutaminase 4"
}

\author{
Nunzianna Doti ${ }^{1}$ \\ 1 Italian National Research Council
}

Potential competing interests: The author(s) declared that no potential competing interests exist.

The paper describe the biochemical characterization of the human transglutaminase 4 (hTG4). Authors found that the hTG4 has a lower enzymatic activity compared to others TGs, prefering slightly acidic pH and reducing conditions. In addition, they showed that its activity can be enhanced by SDS at low concentrations. They also found that the hTG4, unlike hTG2 used as control, has not GTPase binding affinity and the new substrates of the enzyme, identified in this paper, support the hypothesis that the hTG4 can be involved in cell adhesion and migration influencing cancer invasiveness. Finally, they provided a proteomic analysis to check the tissue expression of $\mathrm{Tg} 4$, showing that the enzyme besides the male genital tract, it is physiological present in fetal heart, spleen, salivary glands, colon and urinary bladder.

The manuscript is interesting, rich of results well presented and well discussed.

In my opinion the paper can be accepted in this form. The only advice I can give is that the abstract should be more concise, reducing and / or eliminating the technical information (methodologies used). 